\title{
Influence of Trigonal Warping on Interference Effects in Bilayer Graphene
}

\author{
K. Kechedzhi, ${ }^{1}$ Vladimir I. Fal'ko, ${ }^{1}$ E. McCann, ${ }^{1}$ and B. L. Altshuler ${ }^{1,2}$ \\ ${ }^{1}$ Department of Physics, Lancaster University, Lancaster, LA1 4YB, United Kingdom \\ ${ }^{2}$ Physics Department, Columbia University, 538 West 120th Street, New York, New York 10027, USA
}

(Received 30 January 2007; published 26 April 2007)

\begin{abstract}
Bilayer graphene (two coupled graphitic monolayers arranged according to Bernal stacking) is a twodimensional gapless semiconductor with a peculiar electronic spectrum different from the Dirac spectrum in the monolayer material. In particular, the electronic Fermi line in each of its valleys has a strong $\mathbf{p} \rightarrow$ - $\mathbf{p}$ asymmetry due to trigonal warping, which suppresses the weak localization effect. We show that weak localization in bilayer graphene may be present only in devices with pronounced intervalley scattering, and we evaluate the corresponding magnetoresistance.
\end{abstract}

DOI: 10.1103/PhysRevLett.98.176806

Weak localization (WL), which universally occurs in a broad range of disordered conductors [1,2], is caused by the constructive interference of electron waves circling the same closed path in opposite directions. It is sensitive to the presence of an external magnetic field as manifested in a negative magnetoresistance (MR) at low temperatures.

Usually qualitative features of WL do not depend on the detail of the electronic band structure and crystalline symmetry, with the exception of spin-orbit coupling $[2,3]$. In gapful multivalley semiconductors only the size of the WL effect may depend on the number of valleys and the strength of intervalley scattering [4-6]. The low-field MR, $\Delta \rho(B) \equiv \rho(B)-\rho(0)$, in a two-dimensional electron gas or a thin metallic film $[1,2,4,7]$ in the absence of spinorbit coupling, is characterized by

$$
\Delta \rho(B)=-\frac{s_{\theta} e^{2} \rho^{2}}{2 \pi h} F\left(\frac{B}{B_{\varphi}}\right), \quad B_{\varphi}=\frac{\hbar c}{4 D e} \tau_{\varphi}^{-1} .
$$

Here $F(z)=\ln z+\psi\left(\frac{1}{2}+\frac{1}{z}\right), \psi(z)$ is the digamma function, $\tau_{\varphi}$ is the coherence time, $D$ is the diffusion coefficient, and the integer factor $s_{\theta}$ depends on whether or not states in $n_{v}$ valleys are mixed by disorder. This factor is controlled by the ratio $\theta=\tau_{i} / \tau_{\varphi}$ between the intervalley scattering time $\tau_{i}$ and the coherence time $\tau_{\varphi}$. In materials such as $\mathrm{Mg}$, $\mathrm{ZnO}, \mathrm{Si}, \mathrm{Ge}$ (listed in Table I), where each of the Fermi surface pockets is $\mathbf{p} \rightarrow-\mathbf{p}$ symmetric, intervalley scattering reduces the size of the WL MR from that described by $s_{\infty}=2 n_{v}$ when $\theta=\tau_{i} / \tau_{\varphi} \gg 1$ to $s_{0}=2$ for $\theta \ll 1$.

A more interesting scenario develops in a multivalley semimetal, where the localization properties can be influenced by the absence of $\mathbf{p} \rightarrow-\mathbf{p}$ symmetry of the electronic dispersion within a single valley. Graphene [10-12] in a graphene-based transistor [13-15] represents an example of such a system. In this Letter, we demonstrate how the asymmetry in the shape of the Fermi surface in each of its two valleys determines the observable WL behavior. In contrast with conventional materials (see Table I), WL MR in bilayer graphene is increased by the intervalley scattering, from $s_{\infty}=0$ at $\theta \rightarrow \infty$ to $s_{0}=2$ at $\theta \ll 1$.
PACS numbers: 73.23.-b, 72.15.Rn, 73.43.Qt, 81.05.Uw

Bilayer graphene consists of two coupled graphitic monolayers arranged according to Bernal stacking; see [12] for details of the lattice configuration. Its unit cell contains sites $A, B$ and $\tilde{A}, \tilde{B}$. Sites $A, B$ and $\tilde{A}, \tilde{B}$ belong to the honeycomb lattice in the bottom and top layers, respectively, with sites $B$ being exactly below $\tilde{A}$. The hexagonal Brillouin zone of the bilayer has two inequivalent corners $\mathbf{K}_{+}$and $\mathbf{K}_{-}$[16]. The four branches of its electronic spectrum [17] form one pair split by about $\pm \gamma_{1}$ (the interlayer coupling) and two low-energy branches (formed by states based upon sublattices $A$ and $\tilde{B}$ ) which are degenerate at $\mathbf{K}_{+}$and $\mathbf{K}_{-}$. The low-energy branch can be described [12] using a Hamiltonian,

$$
\begin{aligned}
\hat{H}_{2 L} & =-\frac{1}{2 m}\left[\left(p_{x}^{2}-p_{y}^{2}\right) \sigma_{x}+2 p_{x} p_{y} \sigma_{y}\right]+\hat{h}_{w}, \\
\hat{h}_{w} & =v_{3} \Pi_{z}\left(p_{x} \sigma_{x}-p_{y} \sigma_{y}\right),
\end{aligned}
$$

which acts in the space of four-component wave functions $\Phi=\left[\phi_{\mathbf{K}_{+}, A}, \phi_{\mathbf{K}_{+}, \tilde{B}}, \phi_{\mathbf{K}_{-}, \tilde{B}}, \phi_{\mathbf{K}_{-}, A}\right]$. Here, $\phi_{\xi, \alpha}$ is an electron amplitude on the sublattice $\alpha=A, \tilde{B}$ in the valley $\xi=$ $\mathbf{K}_{+}, \mathbf{K}_{-}, \sigma_{x, y, z}$ and $\Pi_{x, y, z}$ are Pauli matrices acting in sublattice and valley space, respectively [16]. In slightly doped graphene, two disconnected Fermi lines surround the corners of the Brillouin zone.

The first term in Eq. (1) is the leading contribution in the nearest neighbor approximation of the bilayer tight binding model [12]. It includes intralayer hopping $A \rightarrow B$ and

TABLE I. WL factor $s_{\theta}$ in conductors with a multivalley conduction band and negligible spin-orbit coupling. The factor $s_{\theta}$ is specified for two limiting cases, no intervalley scattering $\theta=\tau_{i} / \tau_{\varphi} \rightarrow \infty$, and for fast intervalley scattering $\theta \rightarrow 0$.

\begin{tabular}{clcc}
\hline \hline$n_{v}$ & & $s_{\infty}$ & $s_{0}$ \\
\hline 1 & Mg films [8], ZnO wells [9] & 2 & - \\
2,6 & Si MOSFETs [4,5] & $2 n_{v}$ & 2 \\
2 & Si/SiGe wells [6] & 4 & 2 \\
2 & graphene & 0 & 2 \\
\hline \hline
\end{tabular}


$\tilde{A} \rightarrow \tilde{B}$ (that leads to the dispersion $\epsilon= \pm p v$ near $\mathbf{K}_{ \pm}$in a monolayer) and interlayer $\tilde{A} \rightarrow B$ hopping, and it yields a parabolic spectrum $\epsilon= \pm p^{2} / 2 m$ with $m=\gamma_{1} / 2 v^{2}$. It has been noticed [12] that quasiparticles described by this term are chiral [18]: their plane wave states are eigenstates of an operator $\sigma \mathbf{n}_{2}$ with $\sigma \mathbf{n}_{2}=-1$ for electrons in the conduction band, and $\sigma \mathbf{n}_{2}=1$ for the valence band, where $\mathbf{n}_{2}(\mathbf{p})=(\cos (2 \varphi), \sin (2 \varphi))$ for $\mathbf{p}=(p \cos \varphi, p \sin \varphi)$. For an electron in the conduction band, the plane wave state is

$$
\Phi_{\mathbf{K}, \pm \mathbf{p}}= \pm \frac{e^{ \pm i \mathbf{p x} / \hbar}}{\sqrt{2}}\left(e^{-i \varphi}|\uparrow\rangle_{\mathbf{K}, \pm \mathbf{p}}-e^{i \varphi}|\downarrow\rangle_{\mathbf{K}, \pm \mathbf{p}}\right)
$$

where $|\uparrow\rangle_{\mathbf{K}_{+}, \mathbf{p}}=[1,0,0,0], \quad|\downarrow\rangle_{\mathbf{K}_{+}, \mathbf{p}}=[0,1,0,0] \quad$ and $|\uparrow\rangle_{\mathbf{K}_{-}, \mathbf{p}}=[0,0,1,0],|\uparrow\rangle_{\mathbf{K}_{-}, \mathbf{p}}=[0,0,0,1]$, and the factors $e^{ \pm i \varphi}$ take into account the chirality.

The term $\hat{h}_{w}$ in Eq. (1) originates from a weak direct $A \rightarrow \tilde{B}$ interlayer coupling. This one gives rise to $\mathbf{p} \rightarrow-\mathbf{p}$ asymmetry in the dispersion of electrons. It also leads to a Lifshitz transition in the shape of the Fermi line of the 2D electrons which takes place when $\epsilon_{F} \sim \epsilon_{L}=\frac{1}{4} \gamma_{1}\left(v_{3} / v\right)^{2}$ thus $n_{e}>n_{L} \sim v_{3}^{2} \gamma_{1}^{2} /\left(2 \pi \hbar^{2} v^{4}\right) \sim 10^{11} \mathrm{~cm}^{-2} \quad$ (using $v_{3} / v \sim 0.1$, Ref. [12]). As long as $n_{e}<n_{L}$, the Fermi line in each valley is split into four pockets [12]. For $n_{e}>$ $n_{L}, \hat{h}_{w}$ can be treated as a perturbation leading to a trigonal deformation of a single-connected Fermi line and asymmetry of the electron dispersion inside each valley illustrated in Fig. 1: $\epsilon\left(\mathbf{K}_{ \pm}, \mathbf{p}\right) \neq \epsilon\left(\mathbf{K}_{ \pm},-\mathbf{p}\right)$, though, due to time-reversal symmetry [19], $\epsilon\left(\mathbf{K}_{ \pm}, \mathbf{p}\right)=\epsilon\left(\mathbf{K}_{\mp},-\mathbf{p}\right)$. Existing graphene structures are strongly affected by charges trapped in the underlying substrate or on its surface. Such disorder (as well as the inhomogeneity of the electron density) obscures the intricate details of the electronic Fermi surface at the lowest energies, $\epsilon \ll \epsilon_{L}$. However, the interplay between the two terms in $\hat{H}_{2 L}$

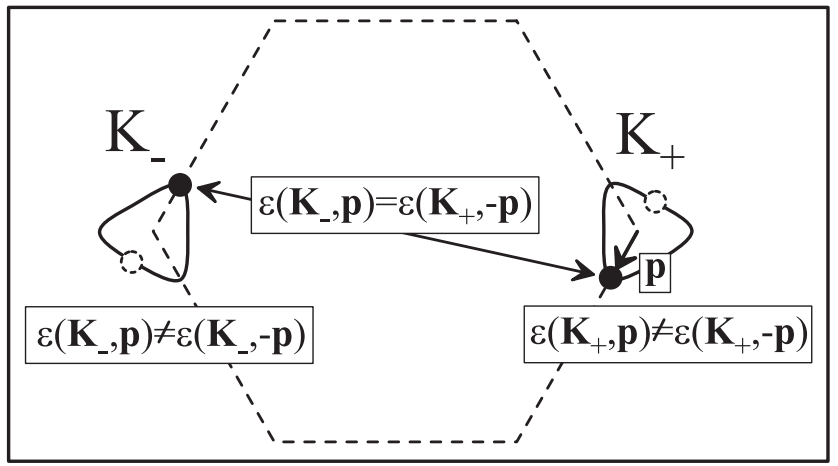

FIG. 1. Fermi lines (solid lines) in the vicinity of two inequivalent valleys $\mathbf{K}_{+}$and $\mathbf{K}_{-}$of the hexagonal Brillouin zone (dashed line). Trigonal warping produces asymmetry of the dispersion at each valley $\epsilon\left(\mathbf{K}_{ \pm}, \mathbf{p}\right) \neq \epsilon\left(\mathbf{K}_{ \pm},-\mathbf{p}\right)$, where momentum $\mathbf{p}$ is determined with respect to the center of the valley, but the effects of warping in the valleys have opposite signs, $\epsilon\left(\mathbf{K}_{ \pm}, \mathbf{p}\right)=\epsilon\left(\mathbf{K}_{\mp},-\mathbf{p}\right)$. The bilayer lattice configuration is described in detail in [12]. resulting in intravalley asymmetry of the electronic dispersion manifests itself in the WL behavior.

The WL correction to conductivity in disordered conductors is a result of the interference of electrons propagating around closed loops in opposite directions [1]. Such interference is constructive in materials without spin-orbit coupling, since electrons acquire exactly the same phase when traveling along two time-reversed paths. It is usually described [1] in terms of the particle-particle correlation function, Cooperon. To evaluate WL in bilayer graphene, we use a Cooperon matrix $C_{\alpha \beta \alpha^{\prime} \beta^{\prime}}^{\xi \mu \xi^{\prime} \mu^{\prime}}$ where subscripts label the sublattice state of incoming $\alpha \beta$ and outgoing $\alpha^{\prime} \beta^{\prime}$ pairs of electrons and superscripts describe the valley state of incoming $\xi \mu$ and outgoing $\xi^{\prime} \mu^{\prime}$ pairs. Following the example of Cooperons for a spin $\frac{1}{2}$ [2], we classify Cooperons as singlets and triplets in terms of sublattice and valley indices $C_{S_{1} S_{2}}^{M_{1} M_{2}}$. For example, $M=0$ is a "valley singlet", $M=x, y, z$ are three "valley-triplet" components; $S=0$ is a "sublattice singlet" and $S=x, y, z$ are "sublattice-triplet" components.

With regards to the sublattice composition of Cooperons in disordered bilayer graphene, only modes $C_{z}^{M}$ (with $S=z$ [20]) are relevant. Indeed, a correlator $C \sim$ $\Phi_{\mathbf{K}_{+}, \mathbf{p}} \Phi_{\mathbf{K}_{-,-p}}$ describing two plane waves, $\Phi_{\mathbf{K}_{+}, \mathbf{p}}$ and $\Phi_{\mathbf{K}_{-},-}$, propagating in opposite directions along a ballistic segment of a closed trajectory, contains only sublatticetriplet terms,

$$
\begin{aligned}
C \sim & \left(|\uparrow\rangle_{\mathbf{K}_{+}, \mathbf{p}}|\downarrow\rangle_{\mathbf{K}_{-},-\mathbf{p}}+|\downarrow\rangle_{\mathbf{K}_{+}, \mathbf{p}}|\uparrow\rangle_{\mathbf{K}_{-},-\mathbf{p}}\right) \\
& -e^{2 i \varphi}|\uparrow\rangle_{\mathbf{K}_{+}, \mathbf{p}}|\uparrow\rangle_{\mathbf{K}_{-,-}-\mathbf{p}}-e^{-2 i \varphi}|\downarrow\rangle_{\mathbf{K}_{+}, \mathbf{p}}|\downarrow\rangle_{\mathbf{K}_{-},-\mathbf{p}},
\end{aligned}
$$

and the terms corresponding to $C_{x, y}^{M}$ disappear after averaging over the direction of momentum, $\left\langle e^{ \pm 2 i \varphi}\right\rangle_{\varphi}=0$.

With regards to the valley composition of relevant Cooperon modes, the symmetry of the electronic dispersion within each valley plays a pivotal role. For a conventional metal [8] or semiconductor [4-6,9], two phases $\vartheta_{\circlearrowright}$ and $\vartheta_{\circlearrowleft}$ acquired by an electron while propagating in a clockwise and anticlockwise direction along the same loop are exactly equal, so that the interference enhances backscattering and leads to WL [1]. Any closed trajectory is a combination of ballistic intervals characterized by momenta $\pm \mathbf{p}_{j}$ (for two directions) and time of flight $t_{j}$. The asymmetry of the electron dispersion in bilayer graphene, due to $\hat{h}_{w}$, generates a phase difference $\delta \equiv \vartheta_{\circlearrowright}-\vartheta_{\circlearrowleft}=$ $\sum \delta_{j}$, where $\delta_{j} \sim\left[\epsilon\left(\mathbf{p}_{\mathbf{j}}\right)-\boldsymbol{\epsilon}\left(-\mathbf{p}_{\mathbf{j}}\right)\right] t_{j}=2 \hat{h}_{w}\left(\mathbf{p}_{\mathbf{j}}\right) t_{j}$. Since $\delta_{j}$ are random uncorrelated, the mean square of $\delta$ accumulated over the time interval $t=\sum_{j} t_{j}$ can be estimated as $\left\langle\delta^{2}\right\rangle \sim\left\langle\left[t_{j} \hat{h}_{w}\left(\mathbf{p}_{\mathbf{j}}\right)\right]^{2}\right\rangle t / \tau$, where $\tau$ is the transport time and $l \equiv v_{F} \tau$ is the mean free path [18]. This determines the relaxation rate,

$$
\tau_{w}^{-1}=\left\{\begin{array}{ll}
\frac{1}{2 \hbar^{2}} \tau\left\langle\operatorname{Tr} \hat{h}_{w}^{2}(\mathbf{p})\right\rangle_{\varphi}=\pi n_{L} l^{2} \tau^{-1}, & \pi n_{L} l^{2}<1 \\
\tau^{-1} & \pi n_{L} l^{2}>1
\end{array},\right.
$$

which suppresses the intravalley Cooperons $C_{z}^{x}, C_{z}^{y}$. We 
estimate that for the recently studied bilayers [11] with $n_{e}=2.5 \times 10^{12} \mathrm{~cm}^{-2}, \tau_{w} \sim \tau$, and $l \sim 0.1 \mu \mathrm{m}$. A similar situation occurs in bilayer structures studied by Gorbachev et al. [21]. Because of time-reversal symmetry $\left[\epsilon\left(\mathbf{K}_{ \pm}, \mathbf{p}\right)=\epsilon\left(\mathbf{K}_{\mp},-\mathbf{p}\right)\right]$ [19], signs of warping in the valleys $\mathbf{K}_{+}$and $\mathbf{K}_{-}$are opposite, so that it does not suppress the intervalley Cooperons $C_{z}^{0}$ and $C_{z}^{z}$.

The above qualitative analysis indicates that strong trigonal warping should be associated with the suppression of WL in bilayer graphene when electrons do not change their valley state upon scattering. To give a quantitative description of this prediction we used the diagrammatic technique and related the WL correction to conductivity to the surviving Cooperon modes [7],

$$
\delta g=\frac{2 e^{2} D}{\pi \hbar}\left(-C_{z}^{z}+C_{z}^{0}-C_{z}^{x}-C_{z}^{y}\right),
$$

where $C(\mathbf{r}, \mathbf{r})$ are the Cooperon propagators taken at coinciding coordinates. For completeness, in Eq. (6) we have retained the intravalley Cooperons $C_{z}^{x, y}$, though they are strongly suppressed by trigonal warping. Following their suppression, the WL correction is determined by the intervalley modes $C_{z}^{0}$ and $C_{z}^{z}$ but, in the absence of intervalley scattering, the contributions of $C_{z}^{0}$ and $C_{z}^{z}$ are equal in magnitude, so that they cancel. Intervalley scattering due to atomically sharp scatterers breaks this exact cancellation and partially restores the WL effect.

A general form of time-reversal-symmetric [19] disorder in bilayer graphene can be represented following the method used in the monolayer graphene studies [22-24]. That is, we determine irreducible representations of the symmetry group of the bilayer crystal formed by $4 \times 4$ matrices acting in the basis of states $|\uparrow\rangle_{\mathbf{K}_{+}, \mathbf{p}},|\downarrow\rangle_{\mathbf{K}_{+}, \mathbf{p}}$, $|\downarrow\rangle_{\mathbf{K}_{-}, \mathbf{p}},|\uparrow\rangle_{\mathbf{K}_{-}, \mathbf{p}}$ and, then, use them to construct the general $t \rightarrow-t$ symmetric disorder $\hat{u}(\mathbf{r})$ :

$$
\begin{aligned}
& \hat{u}=u(\mathbf{r})+\sum \Sigma_{s} \Lambda_{l} u_{s l}(\mathbf{r}) ; \quad s, l=x, y, z ; \\
& \Sigma_{x}=\Pi_{z} \otimes \sigma_{x}, \quad \Sigma_{y}=\Pi_{z} \otimes \sigma_{y}, \quad \Sigma_{z}=\Pi_{0} \otimes \sigma_{z}, \\
& \Lambda_{x}=\Pi_{x} \otimes \sigma_{z}, \quad \Lambda_{y}=\Pi_{y} \otimes \sigma_{z}, \quad \Lambda_{z}=\Pi_{z} \otimes \sigma_{0} .
\end{aligned}
$$

Here, $\vec{\Sigma}=\left(\Sigma_{x}, \Sigma_{y}, \Sigma_{z}\right)$ and $\vec{\Lambda}=\left(\Lambda_{x}, \Lambda_{y}, \Lambda_{z}\right)$ form two mutually commuting algebras $\left[\Sigma_{s_{1}}, \Sigma_{s_{2}}\right]=2 i \varepsilon^{s_{1} s_{2} s_{3}} \Sigma_{s_{3}}$, $\left[\Lambda_{l_{1}}, \Lambda_{l_{2}}\right]=2 i \varepsilon^{l_{1} l_{2} l_{3}} \Lambda_{l_{3}}$, and $[\vec{\Sigma}, \vec{\Lambda}]=0$. Both $\Sigma_{s}$ and $\Lambda_{l}$ invert sign upon time reversal [19], whereas their products remain invariant under $t \rightarrow-t$ transformation. The first term in $\hat{u}$ represents the potential of remote charges which is made short range by screening by $2 \mathrm{D}$ electrons $\left\langle u(\mathbf{r}) u\left(\mathbf{r}^{\prime}\right)\right\rangle \sim u^{2} \delta\left(\mathbf{r}-\mathbf{r}^{\prime}\right)$. It affects electrons in both layers equally and manifests itself through the scattering rate $\tau_{0}^{-1}=\pi \gamma u^{2} / \hbar^{2}$, where $\gamma=m /\left(2 \pi \hbar^{2}\right)$ is the density of states per spin in each valley [7]. Disorder $u_{z z}$ generates a random difference between energies on the $A$ and $\tilde{B}$ sites (bottom and top layers). The two terms containing $u_{x z}$ and $u_{y z}$ originate from fluctuations in hopping and they scatter electrons within each valley, whereas the other terms in $\hat{u}$ take into account intervalley scattering. For simplicity, we assume that different types of disorder are uncorrelated, $\left\langle u_{s l}(\mathbf{r}) u_{s^{\prime} l^{\prime}}\left(\mathbf{r}^{\prime}\right)\right\rangle=u_{s l}^{2} \delta_{s s^{\prime}} \delta_{l l^{\prime}} \delta\left(\mathbf{r}-\mathbf{r}^{\prime}\right)$, and, on average, isotropic in the $x-y$ plane: $u_{x l}^{2}=u_{y l}^{2} \equiv u_{\perp l}^{2}, u_{s x}^{2}=u_{s y}^{2} \equiv$ $u_{s \perp}^{2}$. The corresponding scattering rates $\tau_{s l}^{-1}=\pi \gamma u_{s l}^{2} / \hbar$, where $\tau_{s x}^{-1}=\tau_{s y}^{-1} \equiv \tau_{s \perp}^{-1}$ and $\tau_{x l}^{-1}=\tau_{y l}^{-1} \equiv \tau_{\perp l}^{-1}$ can be combined into the intervalley scattering rate $\tau_{i}^{-1}=$ $4 \tau_{\perp \perp}^{-1}+2 \tau_{z \perp}^{-1}$ and the intravalley rate $\tau_{z}^{-1}=\tau_{z z}^{-1}$, both of which lead to an additional suppression of intravalley modes. Intervalley scattering also leads to the relaxation of $C_{z}^{0}$ although it does not affect the valley-symmetric mode $C_{z}^{z}$. All the scattering mechanisms limit the transport time $\tau^{-1}=\tau_{0}^{-1}+\sum_{s l} \tau_{s l}^{-1}$.

Two low-gap modes $C_{z}^{0}$ and $C_{z}^{z}$ obey the following equations,

$$
\begin{aligned}
{\left[2 \tau_{i}^{-1}+\tau_{\varphi}^{-1}+D \tilde{\mathbf{P}}^{2}-i \omega\right] C_{z}^{0}\left(\mathbf{r}, \mathbf{r}^{\prime}\right) } & =\delta\left(\mathbf{r}-\mathbf{r}^{\prime}\right), \\
{\left[\tau_{\varphi}^{-1}+D \tilde{\mathbf{P}}^{2}-i \omega\right] C_{z}^{z}\left(\mathbf{r}, \mathbf{r}^{\prime}\right) } & =\delta\left(\mathbf{r}-\mathbf{r}^{\prime}\right),
\end{aligned}
$$

where we included an external magnetic field, $\mathbf{B}=\operatorname{rot} \mathbf{A}$ in $\tilde{\mathbf{P}}=\left(i \nabla+\frac{2 e}{c \hbar} \mathbf{A}\right)$, and inelastic decoherence, $\tau_{\varphi}^{-1}(T)$.

Equations (3) and (5) yield the zero field WL correction to the resistivity and the WL MR,

$$
\begin{aligned}
& \frac{\delta \rho(0)}{\rho}=\frac{e^{2} \rho}{\pi h} \ln \left(1+2 \frac{\tau_{\varphi}}{\tau_{i}}\right)+\delta_{0}, \\
& \frac{\Delta \rho(B)}{\rho}=-\frac{e^{2} \rho}{\pi h}\left[F\left(\frac{B}{B_{\varphi}}\right)-F\left(\frac{B}{B_{\varphi}+2 B_{i}}\right)\right]+\delta(B),
\end{aligned}
$$

where $B_{\varphi, i}=\hbar c /\left(4 D e \tau_{\varphi, i}\right)$. Equation (6) gives a complete description of the crossover between two extreme regimes mentioned at the beginning [7]. It also includes small contributions of the suppressed intravalley Cooperons, $\delta_{0}=\left[2 e^{2} \rho /(\pi h)\right] \ln \left(\tau_{\varphi} \tau_{*} /\left[\tau\left(\tau_{*}+\tau_{\varphi}\right)\right]\right) \quad$ and $\quad \delta(B)=$ $-\left[2 e^{2} \rho /(\pi h)\right] F\left[B /\left(B_{\varphi}+B_{*}\right)\right], \quad$ where $\quad \tau_{*}^{-1}=\tau_{w}^{-1}+$ $2 \tau_{z}^{-1}+\tau_{i}^{-1}$ and $B_{*}=\hbar c /\left(4 D e \tau_{*}\right)$. This permits us to account for a possible difference between the warping time $\tau_{w}$ and the transport time $\tau$. According to Eq. (6) WL MR in bilayer graphene sheet disappears as soon as $\tau_{i}$ exceeds $\tau_{\varphi}$, whereas in structures with $\tau_{\varphi}>\tau_{i}$, the result Eq. (6) predicts the WL behavior, as observed in [21]. Such WL MR is saturated at a magnetic field determined by the intervalley scattering time, instead of the transport time as in usual conductors, which provides the possibility to measure $\tau_{i}$ directly.

Also, it is interesting to consider a small device of bilayer graphene with both sizes, length $L$ and width $L_{\perp}$, less than the electron coherence length $\sqrt{D \tau_{\varphi}}$. Since the edge of graphene scatters electrons between $\mathbf{K}_{+}$and $\mathbf{K}_{-}$ valleys, in a wire ("ribbon") with $L_{\perp}<L$ the sample width starts playing the role of the intervalley scattering length, even though intervalley scattering in the bulk of the material may be irrelevant, $L_{i}=\sqrt{D \tau_{i}} \gg L$. In this case, in a wire with conductance $G=G_{\text {classical }}+G_{\mathrm{WL}}+\delta G$ both WL and universal conductance fluctuations (UCFs) 
[25] would be described by the usual results established for the orthogonal symmetry class in disordered systems [25], where UCFs and the WL parts are of the same order, $G_{\mathrm{WL}} \sim-\frac{2}{3} \frac{e^{2}}{h},\left\langle\delta G^{2}\right\rangle^{1 / 2} \sim \sqrt{\frac{8}{15}} \frac{e^{2}}{h}$. In contrast, in a short and wide strip with $L_{\perp}>L$ and no intervalley scattering (either off defects in graphene or contacts), WL in most of the cross section is suppressed by warping, whereas the UCFs (which are immune to such symmetry breaking) are not. Therefore, in a strip $G_{\mathrm{WL}} \sim \frac{e^{2}}{h},\left\langle\delta G^{2}\right\rangle^{1 / 2} \sim 4 \sqrt{\frac{L_{1}}{L}} \frac{e^{2}}{h}$, which not only reflects a larger variance of UCFs in broad samples $\left(L_{\perp} \gg L\right)$, but also the fact that in a valleydegenerate system two valleys give coherent contributions towards the observable conductance fluctuations.

In conclusion, we have shown that $\mathbf{p} \rightarrow-\mathbf{p}$ asymmetry of the electron dispersion in each valley of bilayer graphene leads to unusual (for conventional disordered conductors) behavior of interference effects in electronic transport. Without intervalley scattering, trigonal warping of the electron dispersion near the center of each valley leads to a suppression of weak localization while intervalley scattering restores it. This behavior is expected in experimentally available bilayer graphene structures [11,21], for which $\tau_{w} \sim \tau$. In contrast, the universal conductance fluctuations are not reduced by trigonal warping and may be even stronger in a system without intervalley scattering than in systems with short $\tau_{i}$ : a completely opposite behavior to the WL effect. Such behavior of bilayer graphene is similar to that of monolayer graphene [22], despite the fact that electrons in these two materials have different chiralities and can be attributed different Berry phases: $\pi$ in monolayers [14,22] versus $2 \pi$ in bilayers [26]. More generally, a suppressed weak localization magnetoresistance and its sensitivity to intervalley scattering are specific to all graphitic films independently of their morphology due to lower (trigonal) symmetry of the wave vector $\mathbf{K}$ in the corner of the hexagonal Brillouin zone of a honeycomb lattice crystal. Such a feature of the band structure does not affect the Josephson proximity effect in superconductor-graphene devices. This is because the propagation of a spin-singlet Cooper pair in graphene is related to the valley-symmetric Cooperon $C_{z}^{z}$, whereas Fermi statistics forbid the appearance of the other longliving mode $C_{z}^{0}$ in the superconducting order parameter.

Authors thank V. Cheianov, A. Savchenko, and A. Geim for helpful discussions and the authors of [21] for experimental details.

[1] B. L. Altshuler, D. Khmel'nitzkii, A. I. Larkin, and P. A. Lee, Phys. Rev. B 22, 5142 (1980).

[2] S. Hikami, A. I. Larkin, and Y. Nagaoka, Prog. Theor. Phys. 63, 707 (1980); B. L. Altshuler et al., Sov. Phys. JETP 54, 411 (1981) [Zh. Eksp. Teor. Fiz. 81, 768 (1981)].
[3] P. D. Dresselhaus et al., Phys. Rev. Lett. 68, 106 (1992); G.L. Chen et al., Phys. Rev. B 47, 4084 (1993); J. B. Miller et al., Phys. Rev. Lett. 90, 076807 (2003); A. L. Shelankov, Solid State Commun. 53, 465 (1985).

[4] H. Fukuyama, J. Phys. Soc. Jpn. 49, 649 (1980).

[5] D. J. Bishop, D. C. Tsui, and R. C. Dynes, Phys. Rev. Lett. 44, 1153 (1980); Y. Kawaguchi and S. Kawaji, J. Phys. Soc. Jpn. 48, 699 (1979); R. G. Wheeler, Phys. Rev. B 24, 4645 (1981); R. A. Davies, M. J. Uren, and M. Pepper, J. Phys. C 14, L531 (1981); A. N. Ionov and I. S. Shlimak, JETP Lett. 35, 197 (1982).

[6] R.S. Prasad et al., Semicond. Sci. Technol. 10, 1084 (1995); A. Prinz et al., Thin Solid Films 294, 179 (1997).

[7] We take into account double spin degeneracy of carriers.

[8] G. Bergmann, Phys. Rep. 107, 1 (1984).

[9] A. Goldenblum et al., Phys. Rev. B 60, 5832 (1999).

[10] R. Saito, G. Dresselhaus, and M. S. Dresselhaus, Physical Properties of Carbon Nanotubes (Imperial College Press, London, 1998).

[11] K. S. Novoselov et al., Nature Phys. 2, 177 (2006).

[12] E. McCann and V. I. Fal'ko, Phys. Rev. Lett. 96, 086805 (2006); J. Nilsson et al., Phys. Rev. B 73, 214418 (2006).

[13] K. S. Novoselov et al., Science 306, 666 (2004).

[14] K. S. Novoselov et al., Nature (London) 438, 197 (2005).

[15] Y. Zhang et al., Phys. Rev. Lett. 94, 176803 (2005).

[16] Corners of the hexagonal Brillouin zone are $\mathbf{K}_{ \pm}=$ $\pm\left(\frac{2}{3} h a^{-1}, 0\right)$ ( $a$ is the lattice constant $)$. In the valleys $\mathbf{K}_{ \pm}$, the sublattice spinor $|\uparrow\rangle_{\mathbf{K}_{+}} \equiv[1,0,0,0]$ corresponds to sites $A$, spinor $|\uparrow\rangle_{\mathbf{K}_{-}}=[0,0,1,0]$ to $\tilde{B}$, respectively.

[17] T. Ohta et al., Science 313, 951 (2006).

[18] The disorder-averaged Green's function in the regime $\epsilon_{F} \tau \gg \hbar \quad$ is $\quad G^{R / A}(\mathbf{p}, \epsilon)=\left[\epsilon_{R / A}-\epsilon_{p} \sigma \mathbf{n}_{2}(\mathbf{p})\right] /\left(\epsilon_{R / A}^{2}-\right.$ $\left.\epsilon_{p}^{2}\right)$, where $\epsilon_{R / A}=\epsilon \pm \frac{1}{2} i \hbar \tau_{0}^{-1}$ and $\tau_{0}$ is the scattering time. The dependence $w(\theta) \sim \cos ^{2} \theta$ of the scattering probability off a short-range $A-B$ symmetric potential $u(\mathbf{r})$ on the scattering angle $\theta=\hat{\mathbf{p p}}^{\prime}$ is such that transport and scattering times coincide $\tau=\tau_{0}$. The Drude conductivity is $g=4 e^{2} \gamma D, D=v_{F}^{2} \tau / 2$ [in contrast to monolayer graphene, where $w(\theta) \sim \cos ^{2}(\theta / 2)$ and $\tau=2 \tau_{0}$ ].

[19] In the basis used in this Letter, time reversal $T(W)$ of an operator is given by $T(W)=\Pi_{x} \sigma_{x} W^{*} \Pi_{x} \sigma_{x}$.

[20] Note that $C_{x, y}^{M} \propto\left(|\uparrow\rangle_{\mathbf{K}_{+}, \mathbf{p}}|\uparrow\rangle_{\mathbf{K}_{-,-} \mathbf{p}} \pm|\downarrow\rangle_{\mathbf{K}_{+}, \mathbf{p}}|\downarrow\rangle_{\mathbf{K}_{-,-} \mathbf{p}}\right)$ and $C_{z}^{M} \propto\left(|\uparrow\rangle_{\mathbf{K}_{+}, \mathbf{p}}|\downarrow\rangle_{\mathbf{K}_{-},-\mathbf{p}}+|\downarrow\rangle_{\mathbf{K}_{+}, \mathbf{p}}|\uparrow\rangle_{\mathbf{K}_{-},-\mathbf{p}}\right)$.

[21] R. V. Gorbachev et al., this issue, Phys. Rev. Lett. 98, 176805 (2007).

[22] E. McCann et al., Phys. Rev. Lett. 97, 146805 (2006); H. Suzuura and T. Ando, ibid. 89, 266603 (2002); A. F. Morpurgo and F. Guinea, ibid. 97, 196804 (2006); S. V. Morozov et al., ibid. 97, 016801 (2006).

[23] V. V. Cheianov and V.I. Fal'ko, Phys. Rev. Lett. 97, 226801 (2006).

[24] I. L. Aleiner and K. B. Efetov, Phys. Rev. Lett. 97, 236801 (2006).

[25] B. L. Altshuler JETP Lett. 41, 648 (1985) [Pis'ma Zh. Eksp. Teor. Fiz. 41, 530 (1985)]; P. A. Lee and A. D. Stone Phys. Rev. Lett. 55, 1622 (1985).

[26] At energies $|\epsilon| \ll \epsilon_{L}$ (below the Lifshits transition [12]), the bilayer spectrum in each of the four Fermi surface pockets is linear, and the integral Berry phase $2 \pi$ in bilayer graphene $[11,12]$ is divided into the Berry phases $\pi$ in each of the three side pockets and $-\pi$ in the central one. 